\title{
Laboratory-generated mixtures of mineral dust particles with biological substances: characterization of the particle mixing state and immersion freezing behavior
}

\author{
Stefanie Augustin-Bauditz ${ }^{1, *}$, Heike Wex ${ }^{1}$, Cyrielle Denjean ${ }^{1,4}$, Susan Hartmann ${ }^{1}$, Johannes Schneider ${ }^{2}$, \\ Susann Schmidt ${ }^{2}$, Martin Ebert ${ }^{3}$, and Frank Stratmann ${ }^{1}$ \\ ${ }^{1}$ Leibniz Institute for Tropospheric Research, Permoserstr. 15, 04318 Leipzig, Germany \\ ${ }^{2}$ Max Planck Institute for Chemistry, Hahn-Meitner-Weg 1, 55128 Mainz, Germany \\ ${ }^{3}$ Institute of Applied Geosciences, Schnittspahnstraße 9, 64287 Darmstadt, Germany \\ ${ }^{4}$ National Centre for Meteorological Research, 42 avenue Gaspard Coriolis, 31057 Toulouse, France \\ * Invited contribution by S. Augustin-Bauditz, recipient of the EGU Outstanding Student Poster (OSP) Award 2014.
}

Correspondence to: Stefanie Augustin-Bauditz (augustin@tropos.de)

Received: 2 September 2015 - Published in Atmos. Chem. Phys. Discuss.: 30 October 2015

Revised: 6 April 2016 - Accepted: 9 April 2016 - Published: 4 May 2016

\begin{abstract}
Biological particles such as bacteria, fungal spores or pollen are known to be efficient ice nucleating particles. Their ability to nucleate ice is due to ice nucleation active macromolecules (INMs). It has been suggested that these INMs maintain their nucleating ability even when they are separated from their original carriers. This opens the possibility of an accumulation of such INMs in soils, resulting in an internal mixture of mineral dust and INMs. If particles from such soils which contain biological INMs are then dispersed into the atmosphere due to wind erosion or agricultural processes, they could induce ice nucleation at temperatures typical for biological substances, i.e., above -20 up to almost $0^{\circ} \mathrm{C}$, while they might be characterized as mineral dust particles due to a possibly low content of biological material.

We conducted a study within the research unit INUIT (Ice Nucleation research UnIT), where we investigated the ice nucleation behavior of mineral dust particles internally mixed with INM. Specifically, we mixed a pure mineral dust sample (illite-NX) with ice active biological material (birch pollen washing water) and quantified the immersion freezing behavior of the resulting particles utilizing the Leipzig Aerosol Cloud Interaction Simulator (LACIS). A very important topic concerning the investigations presented here as well as for atmospheric application is the characterization of the mixing state of aerosol particles. In the present
\end{abstract}

study we used different methods like single-particle aerosol mass spectrometry, Scanning Electron Microscopy (SEM), Energy Dispersive X-ray analysis (EDX), and a VolatilityHygroscopicity Tandem Differential Mobility Analyser (VHTDMA) to investigate the mixing state of our generated aerosol. Not all applied methods performed similarly well in detecting small amounts of biological material on the mineral dust particles. Measuring the hygroscopicity/volatility of the mixed particles with the VH-TDMA was the most sensitive method. We found that internally mixed particles, containing ice active biological material, follow the ice nucleation behavior observed for the pure biological particles. We verified this by modeling the freezing behavior of the mixed particles with the Soccerball model (SBM). It can be concluded that a single INM located on a mineral dust particle determines the freezing behavior of that particle with the result that freezing occurs at temperatures at which pure mineral dust particles are not yet ice active.

\section{Introduction}

In the last years a lot of effort has been made to characterize the freezing ability of different aerosol particles, which were thought to be ice nucleating active. Especially mineral dust particles were investigated quite intensively, as they 
were found in ice crystal residues most frequently (Kumai, 1961; DeMott et al., 2003; Pratt et al., 2009). Laboratory measurements indicate that mineral dust particles are efficient ice nucleating particles in a temperature range below $-15^{\circ} \mathrm{C}$ (Murray et al., 2012) or probably only below $-20^{\circ} \mathrm{C}$ (Augustin-Bauditz et al., 2014), where the latter examined droplets in which each contained a single particle of atmospherically relevant sizes. In contrast, atmospheric observations with lidar and radar showed that ice particles also formed at higher temperatures (Seifert et al., 2010; Kanitz et al., 2011; Bühl et al., 2013). It has been assumed in the past that the presence of biological particles like bacteria, fungal spores or pollen is necessary to explain ice nucleation at higher temperatures, as these particles show ice nucleation ability up to temperatures of $-2{ }^{\circ} \mathrm{C}$ (Maki et al., 1974; Morris et al., 2008). Szyrmer and Zawadzki (1997) and later Murray et al. (2012) gave detailed overviews over different types of INP and denominate biological materials as those being ice active at higher temperatures above about $-15^{\circ} \mathrm{C}$. Only recently it became known that biological particles carry small ice nucleating macromolecules (INMs) which are responsible for their freezing ability. These INMs could be e.g., proteins in the case of bacteria (Wolber et al., 1986), fungal spores (Fröhlich-Nowoisky et al., 2015) and material originating from algae contained in the sea surface microlayer (Wilson et al., 2015) or polysaccharides in case of pollen (Pummer et al., 2012). Furthermore, several studies showed that an INM is still ice active when the original carrier is detached or in the case of bacteria non viable (Kleber et al., 2007; Pummer et al., 2012, 2015; Hartmann et al., 2013; Augustin et al., 2013; Fröhlich-Nowoisky et al., 2015). So it is very likely that these INMs are accumulated in the ground, where they come in contact with mineral dust particles. Schnell and Vali (1976) suggested that mineral dust particles may act as inert carriers for biological particles. Pratt et al. (2009) investigated 46 atmospheric cloud ice crystal residues with ATOFMS (Aerosol Time-Of-Flight Mass Spectrometer) and found that $60 \%$ of the dust particles were likely a mixture of biological material and mineral dust. Also Michaud et al. (2014), investigating the residual particles in hail stones, found biological material to be attached to the surface of mineral dust particles. In that context, Kleber et al. (2007) describe that soil organic matter sorbs on mineral surfaces, preserving and maybe even accumulating INMs when being connected to mineral surfaces. Therefore, there is some indication that the ascription of mineral dust to the atmospheric ice nucleating particles (INP) is, at least to a certain extent, due to unnoticed attached ice nucleating biological material (Conen et al., 2011). The question arises how the freezing ability of a mineral dust particle changes when there is some biological material attached to its surface. There are already some laboratory studies which confirm the enhanced freezing ability of soil dust due to the presence of biological material (Conen et al., 2011; O'Sullivan et al., 2014; Tobo et al., 2014). However, the temperature ranges in which the organic fraction of the examined soil dusts was reported to be responsible for the ice activity differed between the studies, extending down to only $-15^{\circ} \mathrm{C}$ for O'Sullivan et al. (2014) (where ice activation observed at lower temperatures was ascribed to mineral dust), but down to even below $-30^{\circ} \mathrm{C}$ for Tobo et al. (2014).

Soil dust is a very inhomogeneous substance and it is very difficult to characterize which of its constituents is responsible for the freezing initiation, particularly as INMs were found to be on the size of a few $10 \mathrm{~nm}$ only (Pummer et al., 2012; Fröhlich-Nowoisky et al., 2015). Also, to quantify the freezing ability of an internal mixture of mineral dust and biological material it is advantageous to know the freezing ability of the individual materials. For this reason in the present study we mixed a well-characterized mineral dust (illite-NX, Hiranuma et al., 2015; Augustin-Bauditz et al., 2014) with well-characterized biological material (birch pollen washing water, Pummer et al., 2012; Augustin et al., 2013) and investigated the immersion freezing ability of the resulting mixed particles, utilizing the Leipzig Aerosol Cloud Interaction Simulator (LACIS). The knowledge of the mixing state of the produced particles is essential for the understanding of the observed freezing abilities. Thus, we applied several methods for characterizing the mixing state of the generated aerosol: single-particle aerosol mass spectrometry, Scanning Electron Microscopy (SEM), Energy Dispersive X-ray analysis (EDX), and a Volatility-Hygroscopicity Tandem Differential Mobility Analyser (VH-TDMA). These methods each use a different approach to characterize the particle mixing state and can therefore be regarded as complementary. One aspect of this study is to compare these characterization methods and to assess their ability to identify the mixing state of a laboratory-generated aerosol. Another important issue is the adequate interpretation of the freezing behavior of the mixed particles. For this purpose the Soccerball model (SBM, Niedermeier et al., 2015) is used. In the following chapters the basics of the SBM, the preparation of the mixture as well as the particle generation will be explained. The applied methods for characterizing the mixing state of the generated aerosol are also described, together with the measurement set-up used for the ice nucleation measurements. Afterwards the results are presented and discussed.

\section{Basics of the Soccerball model}

With the help of the SBM it is possible to describe and parameterize the freezing behavior of different materials. In general it is assumed that freezing is induced by single ice nucleating entities. These entities can be e.g., special sites on the surface of a particle (as it is assumed for mineral dust particles) or, in the case of biological material, single INMs. In any case, the ice nucleating entity has a defined two-dimensional surface area $s_{\text {site. }}$ A specific contact angle $\theta$ is assigned to each ice nucleating entity which determines 
the ice nucleation ability of this particular entity in terms of a nucleation rate coefficient $j_{\text {het }}$ based on classical nucleation theory (Zobrist et al., 2007). The overall contact angle distribution is described by a Gaussian probability density function with a mean value $\mu_{\theta}$ and a standard deviation $\sigma_{\theta}$, with each contact angle $\theta$ occurring with a probability of $p(\theta)$.

$$
p(\theta)=\frac{1}{\sqrt{2 \pi} \sigma_{\theta}} \exp \left(-\frac{\left(\theta-\mu_{\theta}\right)^{2}}{2 \sigma_{\theta}^{2}}\right)
$$

The probability $P_{\text {unfr }}$ of a droplet (which contains an ice nucleating entity) to be unfrozen at a certain temperature $T$ and a certain time $t$ is defined as

$$
\begin{aligned}
P_{\mathrm{unfr}}\left(T, \mu_{\theta}, \sigma_{\theta}, t\right)= & \int_{0}^{\pi} p_{\theta} \exp \left(-j_{\text {het }}(T, \theta) s_{\text {site }} t\right) \mathrm{d} \theta \\
& +\int_{-\infty}^{0} p_{\theta} \exp \left(-j_{\text {het }}(T, \theta=0) s_{\text {site }} t\right) \mathrm{d} \theta \\
& +\int_{\pi}^{\infty} p_{\theta} \exp \left(-j_{\text {het }}(T, \theta=\pi) s_{\text {site }} t\right) \mathrm{d} \theta .
\end{aligned}
$$

As a next step, we consider a population of droplets, with each droplet containing a single particle and all particles having the same size. Naturally, the ice nucleating entities are Poisson distributed over the particle population. This means that each particle contains one, multiple or even none ice nucleating entities. Thus the average number of ice nucleating entities per particle is defined by the expected value of the Poisson distribution $\lambda$ (Hartmann et al., 2013). The ice nucleating probability of each entity is determined by the contact angle distribution. With this, the probability $P_{\mathrm{unfr}, \lambda}$ for droplets to remain unfrozen is given by

$$
P_{\mathrm{unfr}, \lambda}\left(T, \mu_{\theta}, \sigma_{\theta}, \lambda, t\right)=\exp \left(-\lambda\left(1-P_{\mathrm{unfr}}\left(T, \mu_{\theta}, \sigma_{\theta}, t\right)\right)\right) .
$$

Note that $P_{\text {unfr, }, \lambda} \neq P_{\text {unfr }}$. While $P_{\text {unfr }}$ is only valid for droplets which contain an ice nucleating entity, $P_{\mathrm{unfr}, \lambda}$ is valid for the whole droplet population i.e. also for those droplets which do not contain an ice nucleating entity. The ice fraction, which is identical to the freezing probability $P_{\mathrm{fr}, \lambda}$ follows with

$$
f_{\text {ice }}\left(T, \mu_{\theta}, \sigma_{\theta}, \lambda, t\right)=1-P_{\text {unfr }, \lambda}
$$

Equation (4) represents the combination of the original SBM from Niedermeier et al. (2014) and the CHESS model from Hartmann et al. (2013) and was derived in detail in Niedermeier et al. (2015). The average number of ice nucleating entities $\lambda$ is a material and size depending parameter and its determination is dependent on the freezing behavior of the investigated material.

\section{Methods}

\subsection{Materials}

To produce particles consisting of both dust and biological material, first a suspension containing both materials was prepared. For the experiments presented here illite-NX was chosen as the dust component, as this product has been used as a proxy for the natural dust composition found in the atmosphere (Hiranuma et al., 2015, and references therein). Furthermore, the freezing ability of pure illite-NX particles was already investigated with LACIS in a previous study (Augustin-Bauditz et al., 2014). For the illite-NX suspensions $10 \mathrm{~g}$ of illite-NX powder was suspended in $200 \mathrm{~mL}$ of MilliQ water. After shaking the sample it was stored in the refrigerator for about $24 \mathrm{~h}$. During that time large and heavy particles sedimented to the ground. An Eppendorf pipette was used to sample $50 \mathrm{~mL}$ from the top part of the suspension. To determine the concentration of the illite-NX suspension, $10 \mathrm{~mL}$ of the pipetted suspension was dried in a petri dish. A precision balance was used to first determine the weight of the empty petri dish. After drying the suspension the petri dish with the residues was weighted again. This procedure was repeated three times. The mean concentration of the illite-NX suspension is about $0.01 \mathrm{~g} \mathrm{~mL}^{-1}$.

As biological component, washing water from Swedish birch pollen (in the following BPWW) was used. The INM responsible for the freezing ability of BPWW is most likely a polysaccharide (Pummer et al., 2012). The immersion freezing behavior of these INMs was already investigated with LACIS and parameterizations are given in Augustin et al. (2013). The production of the BPWW suspension was done similar to that described in Augustin et al. (2013) but with a lower concentration of $1 \mathrm{~g}$ of pollen grains in $80 \mathrm{~mL}$ of MilliQ water. The birch pollen were mixed with the water and the pollen grains were then removed by filtration (pore size 4-7 $\mu \mathrm{m}$ ) of the resulting suspension. After filtration the concentration of the Swedish birch pollen material in the suspension was determined with the same procedure that was described for the illite-NX suspension above, and it was determined to be about $0.004 \mathrm{~g} \mathrm{~mL}^{-1}$. Thus, after filtration we have about one third of the original mass of pollen in the washing water. This is the same order of magnitude than what was observed by Pummer et al. (2012).

The illite-NX suspension was mixed with the BPWW using $10 \mathrm{~mL}$ of each of the suspensions, which resulted in a dust-bio-mixture to which we refer to in the following as illite-BPWW suspension. Concerning this illite-BPWW suspension, one should be aware of the following facts: a high percentage of the BPWW consists of soluble material which is released when the pollen grains are suspended in water. Thus the BPWW can be viewed as a suspension of INMs (and other larger molecules) in a solution, rather than just a suspension in water. In contrast to that, the dust contains only a small amount of soluble material so its sus- 
pension consists mainly of solid particles and dilute water. These facts are important for the resulting mixing state of the internally mixed illite-BPWW particles and will be referred to again in the next section.

\subsection{Particle generation and characterization}

In Fig. 1 the generation pathway, starting from a suspension and ending with dry particles, is shown schematically. First an atomizer (following the design of TSI 3075) was used to generate droplets from the illite-BPWW suspension as well as from the pure illite-NX and BPWW suspensions. As mentioned above, the BPWW contains a soluble fraction which was distributed in the whole suspension, so it is reasonable to assume that every droplet generated from the illite-BPWW suspension contained at least some soluble biological material, although not necessarily any INMs. Most but maybe not all of these droplets also contained a mineral dust particle. After the atomization the droplets were dried by passing them through a silica gel diffusion dryer. The dry particles were passed through a diffusion charger $(85 \mathrm{Kr})$ to achieve a bipolar charge distribution and afterwards size-selected using a Differential Mobility Analyzer (DMA; Knutson and Whitby, 1975; type "Vienna medium"). In the measurements presented here a mobility diameter of $500 \mathrm{~nm}$ was selected. Due to the use of a cyclone (cut off diameter: $500 \mathrm{~nm}$ ) upstream of the DMA, the fraction of doubly charged particles contained in the aerosol is negligible: this was verified through measurements of the optical particle size distribution done downstream of the DMA using an UHSAS (U1tra High Sensitivity Aerosol Spectrometer, Droplet Measurement Technologies). After size selection the aerosol stream was split and one part was always fed into a Condensation Particle Counter (CPC, TSI 3010) to measure the total particle number concentration. The remaining aerosol was then available for particle characterization (e.g., sampling on filters) and for the Leipzig Aerosol Cloud Interaction Simulator (LACIS). To understand the observed freezing abilities, the knowledge about the mixing state of the generated particles is essential. Considering the above mentioned composition of the generated droplets, we assume that the resulting dry particles were most likely an internal mixture of illite-NX and BPWW material. There might have been also some particles consisting only of BPWW material but we expect no pure illite-NX particles in the generated aerosol. To prove this hypothesis several aerosol characterization methods were applied which will be introduced in the following.

\section{Volatility-Hygroscopicity Tandem Differential Mobility Analyser (VH-TDMA)}

The volatility and hygroscopicity of particles from all three suspensions, the two pure ones and the mixed one were used to infer the particle mixing state. In contrast to mineral dust, biological material is much more volatile and hygroscopic.

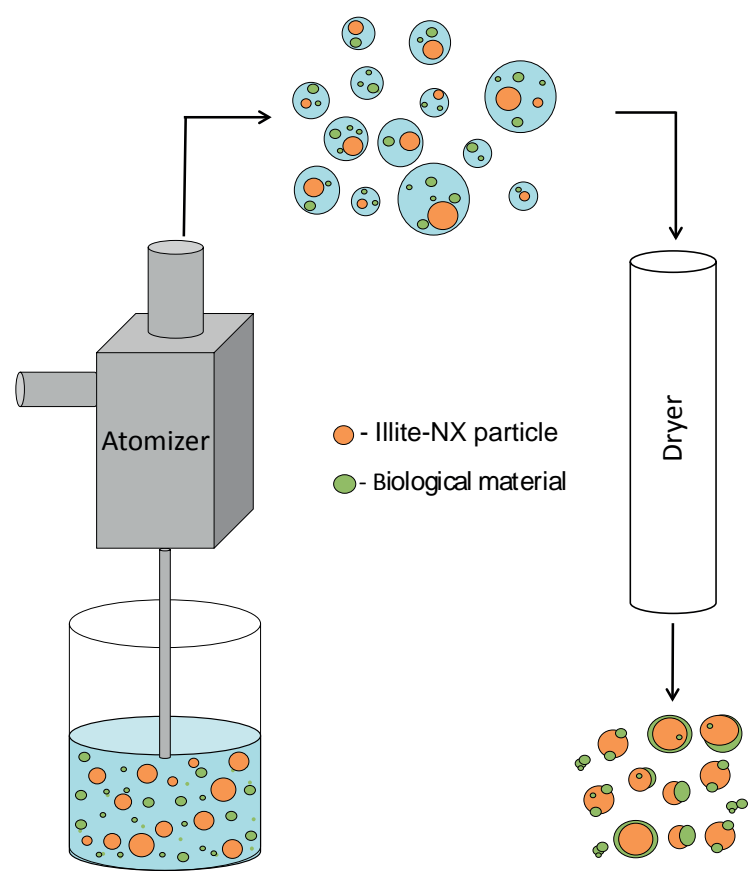

Figure 1. Schematic of the particle generation method used in this study.

Therefore, treatment with heat or humidity will change the size of particles consisting of biological material but likely will have a smaller effect on the mineral dust components. Measurements were performed with a custom-built VHTDMA, which was composed of two DMAs and a CPC (TSI 3010) separated by a volatility (V-mode) and a humidity (Hmode) conditioning device. A mobility diameter of $500 \mathrm{~nm}$, similar to that used for LACIS measurements, was selected with the first DMA. Monodisperse particles were then conditioned either in the thermodenuder section at $300^{\circ} \mathrm{C}$ under dry conditions $(10 \% \mathrm{RH})$ or in the humidity section at $90 \% \mathrm{RH}$ at room temperature $\left(20^{\circ} \mathrm{C}\right)$. The residence times in the conditioning devices were approximately 2 and $4 \mathrm{~s}$ for the $\mathrm{V}$-mode and $\mathrm{H}$-mode, respectively. The resulting particle size distributions obtained after conditioning were measured by the second DMA coupled to the CPC. The second DMA and the humidity section were confined in a temperaturecontrolled box at $20^{\circ} \mathrm{C}$. For the H-mode the sheath air of the second DMA was humidified to $90 \% \mathrm{RH}$. The volatile "growth" factor VGF obtained from the V-mode is

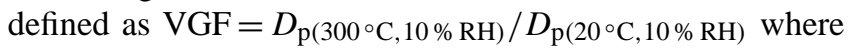
$D_{\mathrm{p}\left(300^{\circ} \mathrm{C}, 10 \% \mathrm{RH}\right)}$ is the measured diameter after heating at $300^{\circ} \mathrm{C}$ and $D_{\mathrm{p}\left(20{ }^{\circ} \mathrm{C}, 10 \% \mathrm{RH}\right)}$ the selected dry mobility diameter at ambient temperature. The hygroscopic growth factor HGF using the H-mode is defined in a similar way: $\mathrm{HGF}=D_{\mathrm{p}\left(20^{\circ} \mathrm{C}, 90 \% \mathrm{RH}\right)} / D_{\mathrm{p}\left(20^{\circ} \mathrm{C}, 10 \% \mathrm{RH}\right)}$ where $D_{\mathrm{p}\left(20{ }^{\circ} \mathrm{C}, 90 \% \mathrm{RH}\right)}$ is the measured diameter at $90 \% \mathrm{RH}$. In this work, the distribution of growth factors refers to the growth factor probability density function (GF-PDF) and was fitted 
as a superposition of distinct Gaussian modes using the TDMAinv algorithm developed by Gysel et al. (2009). From comparing VGF and HGF distributions obtained for the pure and mixed particles, the fraction of internally mixed particles in the aerosol generated from the illite-BPWW suspensions can be derived.

\section{Scanning Electron Microscope (SEM) and Energy Dispersive X-ray analysis (EDX)}

In cooperation with the Leibnitz institute of surface modification in Leipzig (IOM) and the Technical University Darmstadt filter samples generated from the illite-BPWW suspension as well as from the pure illite-NX and BPWW suspensions were imaged by SEM. Analysis at the IOM was performed in a ULTRA 55, Carl Zeiss SMT, (Oberkochen, Germany) and in Darmstadt in a FEI Quanta 200 FEG Environmental Scanning Electron Microscope (Eindhoven, the Netherlands).

By SEM we intended to examine visual differences between particles generated from the two pure substances and from the illite-BPWW suspensions to get more information about the exact mixing-state of the illite-BPWW aerosol, i.e., if the sample can be described as an external mixture of the two components, as an agglomeration of the two components or as an internal mixture. Additionally, at the Technical University Darmstadt the pure samples and the particles from the illite-BPWW suspension were analyzed by EDX (Phoenix EDAX, Tilburg, the Netherlands). With this technique the elemental composition of individual particles is determined. In order to prevent an influence of the sampling substrate on the chemical results (carbon signal from the filters) for these analyses the particles were sampled on Boron substrates.

\section{Single Particle Laser Ablation Time-of-flight mass spectrometer (SPLAT)}

Finally we also investigated the mixing state of the illiteBPWW aerosol via single particle mass spectrometry. Here, the instrument SPLAT (Kamphus et al., 2008) of the Max Planck Institute for Chemistry, Mainz, was used. For these experiments, particles were generated with the set-up described above and then examined in the SPLAT immediately after generation. This was done at first separately for an illite suspension and for BPWW, and then for the mixed particles generated from the illite-BPWW suspension. In the SPLAT, single particles are hit by a laser pulse which vaporizes and ionizes the compounds of the particle. A bipolar time-of-flight mass spectrometer is used to detect the ions. The composition and thereby the mixing state of the individual particles can then be inferred from their mass spectra.

\subsection{Freezing experiments}

For the freezing experiments the laminar flow tube LACIS was utilized. In the inlet section of LACIS, the aerosol flow is combined isokinetically with a humidified sheath air flow such that the aerosol forms a beam of approximately $2 \mathrm{~mm}$ in diameter along the center line of the flow tube. Supersaturated conditions are achieved by cooling the tube walls, and result in activation of the particles to droplets, with each droplet containing one size segregated particle. Further downstream of the flow tube, these droplets then may freeze due to further cooling. For detailed information on the operation mode of LACIS see Stratmann et al. (2004) and Hartmann et al. (2011).

At the outlet of LACIS, TOPS-Ice (thermally stabilized optical particle spectrometer, Clauss et al., 2013) is used to discriminate between frozen and unfrozen droplets, and to quantify the fraction of frozen $\operatorname{droplet}\left(f_{\text {ice }}\right.$, number of frozen droplets divided by the total number of frozen and unfrozen droplets). In the investigations presented here, $f_{\text {ice }}$ was determined in the temperature range between $\sim-17$ and $-40^{\circ} \mathrm{C}$.

\section{Measurement results}

In the following we will first describe the results concerning the determination of the mixing state of the generated aerosol (Sect. 4.1). Afterwards, the results of the immersion freezing experiments will be discussed (Sect. 4.2).

\subsection{Characterization of the particle mixing state using different methods}

Several methods were applied to characterize the mixing state of the generated illite-BPWW aerosol. These were already introduced in Sect. 3.2 and their results will be discussed in the following.

\subsubsection{VH-TDMA}

The HGF and VGF distributions of the different particle types which were obtained from the VH-TDMA measurements are shown in Fig. 2. The values for the mean and standard deviation of the different curves are given in Table 1. For the pure illite-NX particles (orange line) almost no change in size was observed for both treatments, which results in mean growth factors of approximately 1 . This confirms that the illite-NX contained no or only very little amounts of volatile or soluble material. In contrast to that, particles generated from the BPWW suspension (green line) showed a significant change in size for both treatments which lead to HGF and VGF of 1.38 and 0.56 , respectively. The results for the particles generated from the illite-BPWW suspension (purple line) showed HGF and VGF values which are between those for the pure substances. It is also obvious that only one mode is present and that this mode shows no (for the VGF) 

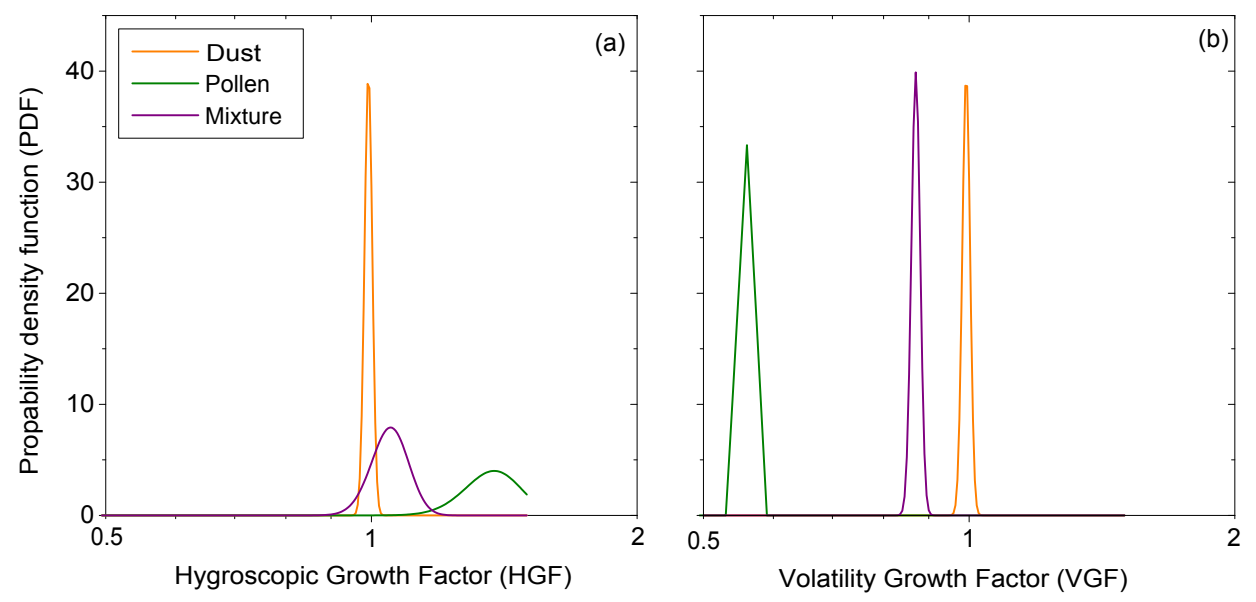

Figure 2. (a) Probability density function (PDF) of the hygroscopic growth factor (HGF) determined from the VH-TDMA measurements at $90 \%$ relative humidity. (b) PDF of the volatile "growth" factor (VGF) determined from the VH-TDMA measurements at $300{ }^{\circ} \mathrm{C}$.

Table 1. Mean value and standard deviation of the HGF and VGF Gaussian distribution for the different heated (V) and humidified (H) particle types. The selected size prior to the treatment was $500 \mathrm{~nm}$.

\begin{tabular}{llrr}
\hline & Suspension & Mean & Standard deviation \\
\hline \multirow{4}{*}{ HGF } & illite-NX & 0.99 & 0.01 \\
& BPWW & 1.38 & 0.07 \\
& illite-BPWW & 1.05 & 0.05 \\
\hline \multirow{4}{*}{ VGF } & illite-NX & 0.99 & 0.01 \\
& BPWW & 0.56 & 0.02 \\
& illite-BPWW & 0.87 & 0.01 \\
\hline
\end{tabular}

or only little (for the HGF) overlap with the pure BPWW material. This suggests that nearly all particles from the illiteBPWW suspension consisted of both, illite-NX and BPWW material. In other words: all particles were internally mixed. Furthermore, the HGF values can be used to determine the hygroscopicity parameters $\kappa$ of the different particle types (Petters and Kreidenweis, 2007). These $\kappa$ values are 0, 0.176 and 0.017 for pure illite-NX, pure BPWW and internally mixed particles, respectively, determined from average HGF. For the $\kappa$ of the internally mixed particles the simple mixing rule $\kappa_{\text {mix }}=\sum_{i} \epsilon_{i} \kappa_{i}$ can be applied (Petters and Kreidenweis, 2007). Here, $\epsilon_{i}$ depicts the amount of material $i$ which has a $\kappa$ value of $\kappa_{i}$. With this it is possible to calculate the average volume fraction of BPWW material on the internally mixed particles, which was found to be $9.7 \%$. Assuming spherical illite-NX particles surrounded by a smooth layer of BPWW material, the BPWW layer thickness would be $8 \mathrm{~nm}$.

\subsubsection{SEM and EDX}

The typical routine for the EDX based identification of particles as internal/external mixtures is the use of elemental ra- tios of main elements of the pure components (within the individual particles in the mixed sample) as classification criteria. In this study the classification of the analyzed particles is based on the determined carbon / silicon $(\mathrm{C} / \mathrm{Si}) \mathrm{ra}-$ tio. The choice of the boundary values of the elemental ratios for the classification as internal respectively external mixture depends on the detection limit and uncertainty of the EDX measurement. Marginal carbon and silicon signals (close to detection limit) are often observed in SEM-EDX measurements. In this way only particles with a $\mathrm{C} / \mathrm{Si}$ ratio (based on net count rate) between 0.1 and 10 can be classified as internal illite/BPWW mixtures. As EDX analysis is limited to major and minor elements, internal mixtures cannot be identified when one component is only present in traces.

Following this scheme all particles from the illite-BPWW suspension would have been classified as pure illite samples. Including the morphological data from the SEM measurements it becomes obvious that the particles from the illiteBPWW suspension are neither morphologically (secondary electron images, see Fig. $3 b$ and c) nor chemically (EDX) discriminable from the pure illite particles by SEM analysis. Furthermore, nearly none of the viscous droplets (Fig. 3a), which would indicate the presence of pure BPWW particles could be observed in the SEM images of the illite-BPWW particles (145 particles were counted from which only 1 showed a shape similar to the pure BPWW particles).

With respect to the limitations of EDX analysis it can be concluded that the relative abundance of the BPWW residuals within the mixed particles is less than (maximum) $2 \mathrm{wt} \%$. As the morphological surface features of the illite particles are still visible in the illite-BPWW suspension, a surface coating, which is thicker than a few tens of nanometers, can be excluded. This is consistent with the results from the VHTDMA measurements, where a layer thickness of $8 \mathrm{~nm}$ was estimated. As the residual carbonaceous particles (which will contain the INMs) from the BPWW will be sited on the sur- 

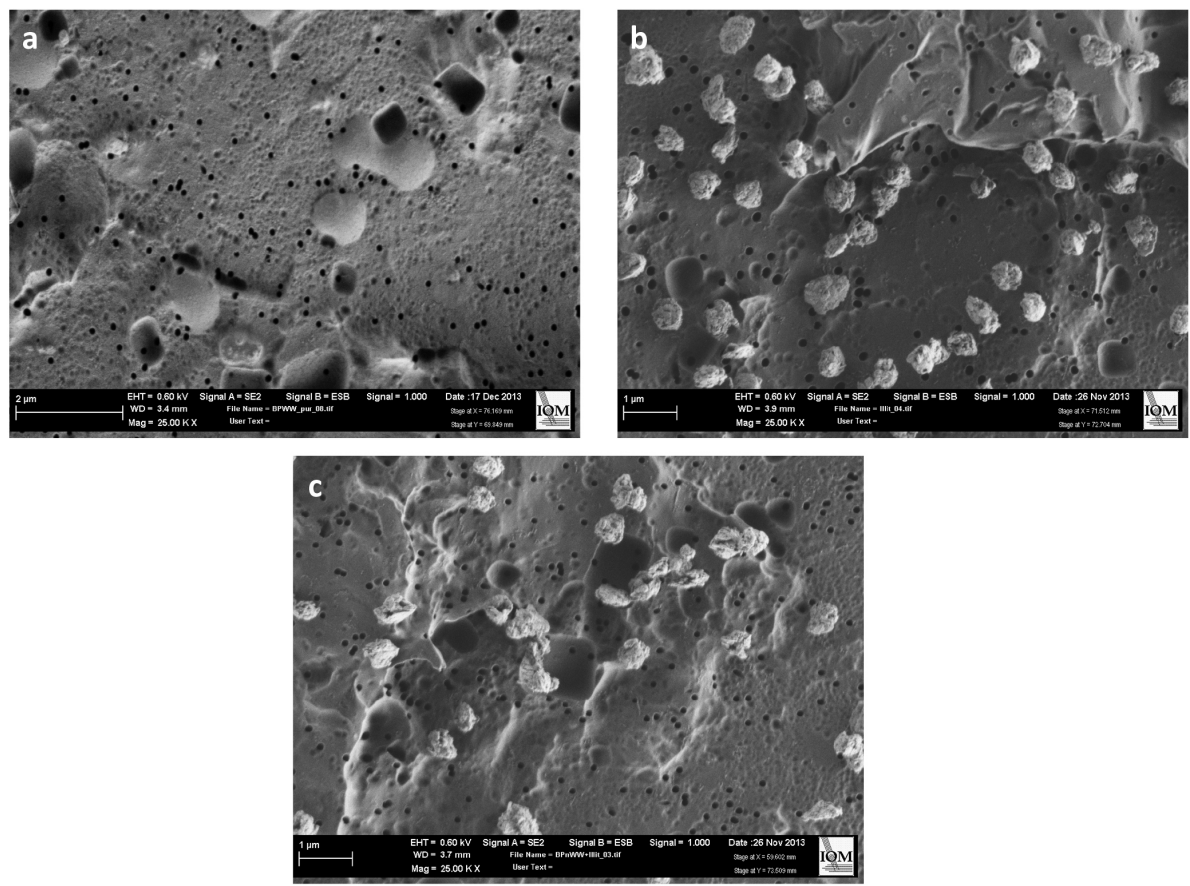

Figure 3. Examples of SEM images of the different suspensions. (a) BPWW, (b) illite-NX, (c) illite-BPWW.

face of the illite grains, it is highly likely that they are present either as very thin film or as small "isles" at the surface of the illite particles.

\subsubsection{Single particle mass spectrometry}

For the illite-BPWW suspension 549 mass spectra were measured with the SPLAT, 150 of which had to be discarded due to insufficient ion count rate or wrong mass calibration. The remaining 399 mass spectra were used for the further analysis.

To decide whether a particle is an internal mixture or a pure particle, the peak intensity ratio of selected marker peaks $(\mathrm{Na} /(\mathrm{Na}+\mathrm{SiO}))$ was used (histograms of this ratio as well as examples of mass spectra for the pure as well as for the mixed particles are shown in Fig. 4).

The experiments with the pure particles showed that values of this ratio between 0 and 0.1 occurred only for pure illite, while ratios larger than 0.65 were only observed for pure BPWW particles. Thus, particles with ratios between these threshold values are likely mixtures of both components.

328 (around $82 \%$ ) out of the 399 spectra showed a ratio $\mathrm{Na} /(\mathrm{Na}+\mathrm{SiO})$ between 0.1 and 0.65 , indicating internal mixtures of illite-NX and BPWW material. Only 59 particles (14\%) appeared to be pure illite, and $16(4 \%)$ appeared to be pure BPWW in the SPLAT.

The uncertainties of this method can be estimated as follows: $25 \%$ uncertainty originates from the amount of useful mass spectra per measurement (about $25 \%$ of the mass spectra recorded in each experiment had to be discarded out due to insufficient ion count rate or wrong mass calibration). Additionally, $37 \%$ of the pure illite values as well as $44 \%$ of the pure BPWW values have intensity ratios in the intermediate range from 0.1 to 0.65 . These uncertainties result in a possible underestimation of externally mixed particles of up to about a factor of 1.6 for pure illite and 1.8 for pure BPWW. This "worst case scenario" would lead to values of $24 \%$ pure illite particles and $7 \%$ pure BPWW particles, which still means that $69 \%$ of the particles are internally mixed.

On the other hand, as discussed above, it is rather unlikely to find pure illite-NX particles in the aerosol generated from the illite-BPWW suspension. But as the VH-TDMA measurements as well as the SEM images and the EDX analysis suggest that the amount of BPWW material on the particles might be rather small, it can not be ruled out that the SPLAT can not detect such small amounts of biological material and thus the amount of internally mixed particles is underestimated.

It is obvious that the analysis of the mixing state of the generated illite-BPWW aerosol is not trivial and that it is bound to the limitations of the applied instruments. What we learned from these investigations is that, especially for particles with only small amounts of biological material, the detection of this biological material is difficult or even not possible. Nevertheless, all methods showed (albeit some only indirectly) that a significant fraction, if not all, of the generated particles consisted of both biological material and dust and are internally mixed particles. 

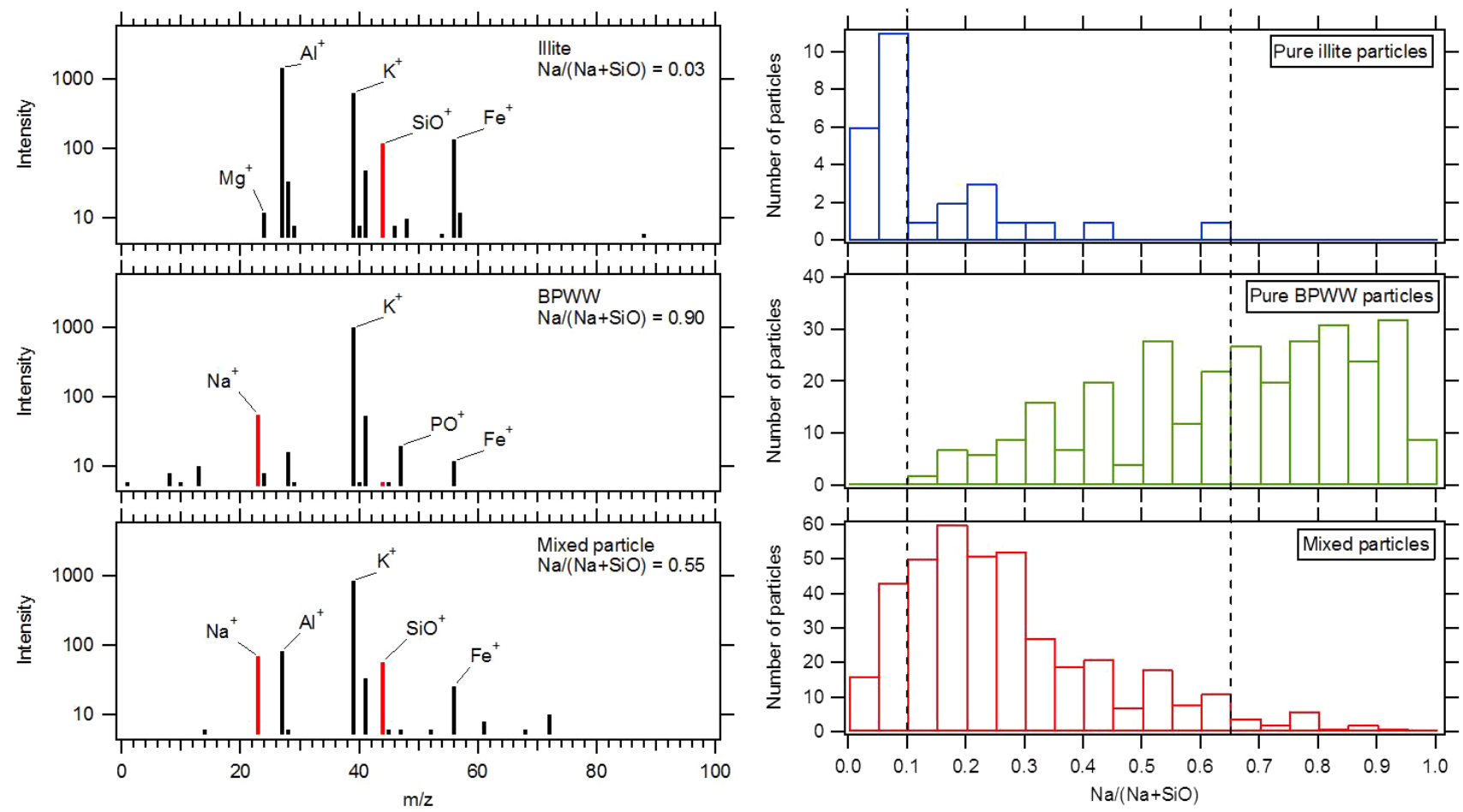

Figure 4. (left) Examples of single particle mass spectra of a pure illite particle, a pure BPWW particle, and a mixed particle. The marker peaks $\mathrm{Na}^{+}(m / z, 23)$ and $\mathrm{SiO}^{+}(m / z 44)$ are highlighted. The values for $\mathrm{Na} /(\mathrm{Na}+\mathrm{SiO})$ are given for each particle. (right) Histograms of the ratio $(\mathrm{Na} /(\mathrm{Na}+\mathrm{SiO})$ for all particles. The dashed lines indicate the boundaries for pure illite $(<0.1)$ and for pure $\mathrm{BPWW}(>0.65)$.

\subsection{Immersion freezing experiments}

In Fig. 5 the results of the immersion freezing experiments of the particles generated from the illite-BPWW suspension are shown (purple symbols). For a better understanding of the freezing behavior of the mixed particles it is necessary to first understand the freezing behavior of the pure materials. Therefore, results of measurements from the pure $500 \mathrm{~nm}$ illite-NX and BPWW particles are shown in Fig. 5, as orange and green symbols, respectively. The freezing ability of illite-NX particles generated from suspension was compared to the freezing ability of dry generated illite-NX particles of the same size $(500 \mathrm{~nm})$ as presented in Augustin-Bauditz et al. (2014) (filled and open orange squares in Fig. 5, respectively). The ice nucleation ability of the wet generated particles was only slightly below that of the dry generated ones. Concerning the BPWW measurements shown here, we should mention that we had to use another birch pollen batch than in Augustin et al. (2013) as the one used in the former study was used up. Similarities and differences between these two batches are addressed further down in the manuscript.

The comparison between the freezing behavior of the particles generated from the illite-BPWW suspension and the pure substances indicates that it is possible to explain the immersion freezing behavior of the mixture by the freezing abilities of the pure substances. Down to roughly $-30^{\circ} \mathrm{C}$, the temperature range at which the first steep increase is observed, and also the subsequent course of the ice fraction, are identical to those of pure BPWW particles. The second increase in the frozen fraction below $-30^{\circ} \mathrm{C}$, from roughly 0.2 to above 0.3 , occurs in a temperature range for which the ice nucleation is observed for pure illite-NX particles. At about $-38^{\circ} \mathrm{C}$ the homogeneous freezing sets in. The solid lines in Fig. 5 represent modeled ice fractions for the different particle types. The next section will describe how these curves were obtained for the pure particles and how they can be combined to describe the ice nucleation behavior of the internally mixed particles.

\section{Theoretical description and discussion}

On the first view it seems to be fairly clear just from the three experimental data sets in Fig. 5 that the freezing behavior of the mixed particles can be explained by the freezing behaviors of the pure substances. Nevertheless, this conclusion has to be checked by adequate modeling. For this purpose we decided to use the Soccerball model (SBM, Niedermeier et al., 2015, see Sect. 2). We will first introduce the parameterizations for the pure substances, and afterwards the ice nucleation of the mixed illite-BPWW particles will be modeled. 


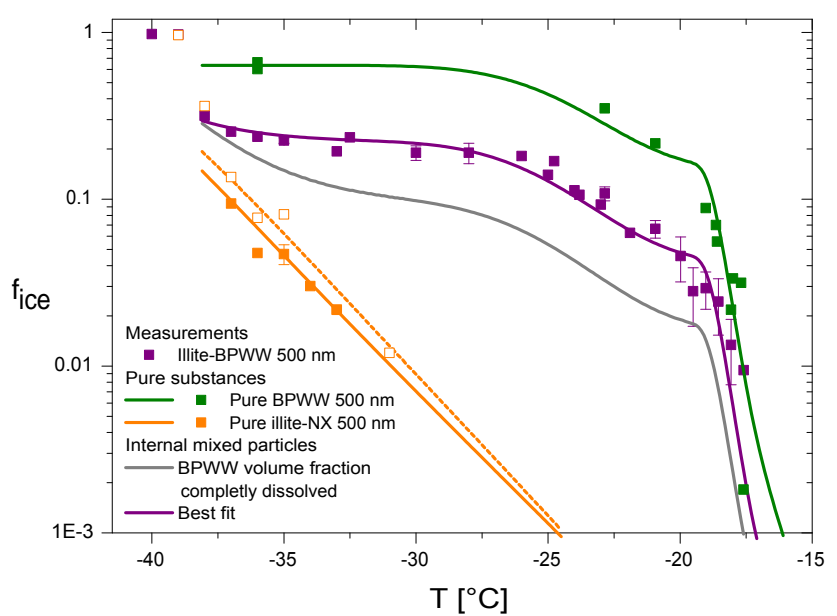

Figure 5. Results of the immersion freezing experiments of particles with a mobility diameter of $500 \mathrm{~nm}$ generated from the illiteBPWW-suspension as well as from the pure illite-NX and BPWW particles (purple, orange and green symbols, respectively). Filled orange squares: illite-NX particles from suspension, open orange squares: illite-NX particles from dry generation. Error bars are the standard deviation of the experiments and were obtained for temperatures with at least three measurements. The green, the dashed orange and the straight orange line represent the freezing ability of pure BPWW, dry generated illite-NX and wet generated illiteNX particles, respectively ( $500 \mathrm{~nm}$ mobility diameter) based on the SBM parameters determined from measurements with the pure substances. The grey line represents an extreme case where all the available BPWW material (9.7\% volume fraction) on the mixed particles has been dissolved in the droplet. The purple line represents the freezing behavior of internally mixed particles which was fitted to the measured data.

\subsection{Immersion freezing properties of BPWW particles}

For the freezing behavior of BPWW (green line in Fig. 5) it was observed that the ice fraction reaches a saturation range below one (Augustin et al., 2013), which means that at a certain temperature no further increase in ice fraction with decreasing temperature is observed. It is known that single INMs are responsible for the freezing behavior of the BPWW material (Pummer et al., 2012). The occurrence of a saturation range below one implies that not all of the particles generated from a BPWW suspension contain an INM (a similar behavior was observed for Snomax in Hartmann et al., 2013). In those cases the average number of INM per droplet $(\lambda)$ can be directly calculated from the ice fraction $f_{\text {ice }}^{*}$ observed in the saturation range, with: $\lambda=-\ln \left(1-f_{\text {ice }}^{*}\right)$.

In Augustin et al. (2013) it was possible to describe the $\lambda$ value of the BPWW by both a linear particle volume dependence and a linear particle surface area dependence, where a surface area dependence yielded a slightly better regression coefficient (surface area: $r=0.9918$, and volume: $r=$ 0.9275). This was somehow surprising as it was expected that the BPWW particles are fully soluble and thus should show a clear linear volume dependence (as for example in the case of Snomax; Hartmann et al., 2013). In Augustin et al. (2013) it was speculated that the BPWW contains some slowly dissolving material and that the generated particles may need more time than the few seconds they have in LACIS to dissolve completely, which would explain the surface dependence of $\lambda$.

It should be mentioned here that the Swedish birch pollen washing water which was used in this study contains two different types of INM (called INM- $\alpha$ and INM- $\beta$ ), which are internally mixed (Augustin et al., 2013). So for the BPWW used in this study Eq. (3) changes to

$$
\begin{aligned}
P_{\mathrm{unfr}, \lambda}^{\mathrm{BPWW}}\left(T, \mu_{\theta}, \sigma_{\theta}, \lambda, t\right)=\exp ( & -\lambda_{\alpha}\left(1-P_{\mathrm{unfr}, \alpha}\right) \\
& \left.-\lambda_{\beta}\left(1-P_{\mathrm{unfr}, \beta}\right)\right) .
\end{aligned}
$$

The two different types of INM are the reason for the shoulder of the BPWW ice fraction curve at around $-20^{\circ} \mathrm{C}$, where the increase at temperatures above and below this shoulder is caused by the more and the less ice active type of INM being active, respectively. The values for $\mu_{\theta}$ and $\sigma_{\theta}$ for both INM types contained in the BPWW were taken from Augustin et al. (2013) and are given in Table 3.

The size of a single INM was estimated to be $10 \mathrm{~nm}$ (Pummer et al., 2012). Assuming spherical INMs this leads to a $s_{\text {site }}$ of $3.14 \times 10^{-16} \mathrm{~m}^{2}$. As explained above, the $\lambda$ values of INM- $\alpha$ and INM- $\beta$ can be described by both a linear volume dependence and a linear surface area dependence. The relations between $\lambda$ and particle volume for INM- $\alpha$ and INM- $\beta$ can be described as follows:

$\lambda_{\alpha}=6.76 \times 10^{18} \mathrm{~m}^{-3} \times D_{\mathrm{p}}^{3}$ and

$\lambda_{\beta}=1.31 \times 10^{18} \mathrm{~m}^{-3} \times D_{\mathrm{p}}^{3}$.

When assuming a surface area dependence the relations are

$\lambda_{\alpha}=3.30 \times 10^{12} \mathrm{~m}^{-2} \times D_{\mathrm{p}}^{2}$ and

$\lambda_{\beta}=6.65 \times 10^{11} \mathrm{~m}^{-2} \times D_{\mathrm{p}}^{2}$.

The respective values for a $500 \mathrm{~nm}$ particle are given in Table 2. These values differ a little from the respective values given in Augustin et al. (2013). The reason for that is that a new birch pollen batch had to be used for the here presented measurements. This includes both measurements of pure BPWW and those for mixed illite-BPWW particles. It is not surprising that due to natural variability the number of INMs produced per pollen grain or per mass of pollen varies. As a result, the number of INMs per particle also differs from batch to batch. But the ice nucleation properties $\left(\mu_{\theta}\right.$ and $\left.\sigma_{\theta}\right)$ which were determined for the old pollen batch can be used to model the ice nucleation behavior of particles produced from the new batch, as seen by the good agreement between measured and modeled data for BPWW shown in Fig. 5. This is a strong indication for the fact that the two types of INMs in the new batch as such are the same than those in the formerly used batch. 
Table 2. Summary of the $\lambda$ values for $500 \mathrm{~nm}$ particles of different composition. They were calculated with the given relations in Sect. 5.1 for BPWW particles and Sect. 5.2 for illite-NX particles. For the calculation of the values of case (b) it was assumed that the soluble volume fraction of the BPWW material in the internally mixed particle is approximately $9.7 \%$. The values for case (c) were estimated by fitting Eq. (7) to the measured data with $\lambda_{\alpha}, \lambda_{\beta}$ and $\lambda_{\text {illite }}$ being the fit parameters, where the relation between $\lambda_{\alpha}$ and $\lambda_{\beta}$ remains the same as derived from the measurements of the pure BPWW. The $\lambda$ values shown here are only valid for the respective particles generated for the measurements in this study.

\begin{tabular}{lrrr}
\hline $500 \mathrm{~nm}$ particle & $\lambda_{\text {illite }}$ & $\lambda_{\alpha}$ & $\lambda_{\beta}$ \\
\hline pure illite & 0.813 & - & - \\
pure BPWW (surface dependent) & - & 0.825 & 0.166 \\
Case (b) (Fig. 6) & 0.759 & 0.082 & 0.016 \\
Case (c) (Fig. 6) & 0.511 & 0.206 & 0.041 \\
\hline
\end{tabular}

\subsection{Immersion freezing properties of illite-NX particles}

In contrast to the BPWW particles we did not observe a saturation range for the frozen fraction for the illite-NX particles (Augustin-Bauditz et al., 2014). But, due to the lower ice nucleating ability, it is plausible that homogeneous ice nucleation, which is dominant for $T<-38^{\circ} \mathrm{C}$, perhaps masks a potential leveling off of the frozen fraction curves. Therefore, we also used the presented procedure for representing the ice nucleating ability of the pure illite-NX particles (orange lines in Fig. 5). To do so, the following assumptions were made. First, the $\lambda$ value of illite-NX is assumed to be smaller than the determined $\lambda$ value for feldspar given in Niedermeier et al. (2015). This is a reasonable assumption as K-feldspar was observed to be the most ice active mineral dust found so far (Atkinson et al., 2013; Augustin-Bauditz et al., 2014). Second, we assume that $\lambda$ is directly correlated to the particle surface area, as for mineral dust it is assumed that the ice nucleating entities are special sites on the surface of the particle. This correlation between $\lambda$ and particle surface area was already observed for feldspar particles (Niedermeier et al., 2015). Due to these assumptions we could distinctly narrow the range of the possible $\lambda$ parameter. For a $s_{\text {site }}$ of $10^{-14} \mathrm{~m}^{2}$ as used in Niedermeier et al. (2015), the best fit between measured and modeled data was obtained for $\lambda_{\text {illite }}=3.25 \times 10^{12} \mathrm{~m}^{-2} \times D_{\mathrm{p}}^{2}$. For a $500 \mathrm{~nm}$ particle the $\lambda_{\text {illite }}$ would be 0.813 (see Tab. 2). The resulting $\mu_{\theta}$ and $\sigma_{\theta}$ for both wet and dry generated particles are given in Table 3 . For the calculations in the next section, the parameters of the wet generated particles were used, as the illite-BPWW particles were also generated from a suspension.

\subsection{Immersion freezing properties of illite-BPWW particles}

As already indicated, the freezing behavior of the mixed particles appears as superposition of the single substances'
Table 3. Soccerball model parameters used for the calculations shown in Fig. 5. The values for $\mu_{\theta}$ and $\sigma_{\theta}$ are determined from measurements with the pure substances. The BPWW parameters were directly taken from Augustin et al. (2013). The parameters for illite-NX particles (wet and dry generation) were calculated within this study.

\begin{tabular}{lrr}
\hline & $\mu_{\theta}$ [rad] & $\sigma_{\theta}$ [rad] \\
\hline pure illite-NX (dry generation) & 1.903 & 0.274 \\
pure illite-NX (wet generation) & 2.022 & 0.315 \\
pure BPWW (INM- $\alpha$ ) & 1.016 & 0.0803 \\
pure BPWW (INM- $\beta$ ) & 0.834 & 0.0005 \\
\hline
\end{tabular}

freezing behaviors, and the most ice active ice nucleation entity within the particle will dominate the freezing process. For the $500 \mathrm{~nm}$ illite-BPWW particles considered here the INMs of the BPWW material are the dominant ice nucleation entities in the temperature range between -17 and $-30^{\circ} \mathrm{C}$. At this point, it is worth mentioning that although we assume that every particle contains some material from the BPWW, not every particle will contain an INM, as already pure $500 \mathrm{~nm}$ BPWW particles did not all contain an INM. Hence in some illite-BPWW particles, it will be the illite which induces droplet freezing.

In the following, we model the ice nucleation behavior of the illite-BPWW particles based on the SBM parameters $\left(\mu_{\theta}\right.$ and $\sigma_{\theta}$ from Table 3 ) for the pure substances. For the illiteNX component the parameters of the wet-generated particles were used.

First we consider that all particles are internally mixed (see discussion in Sect. 4.1). As independent probabilities are multiplicative, the $P_{\text {unfr, mix }}$ is calculated as follows:

$P_{\text {unfr,mix }}\left(T, \mu_{\theta}, \sigma_{\theta}, \lambda, t\right)=P_{\text {unfr }, \lambda}^{\text {illite }} \times P_{\text {unfr }, \lambda}^{\mathrm{BPWW}}$.

With that the ice fraction can be determined as follows:

$f_{\text {ice }}\left(T, \mu_{\theta}, \sigma_{\theta}, \lambda, t\right)=1-P_{\text {unfr, mix }}$.

Equation (7) now represents the freezing behavior of particles, which consist of both illite-NX and BPWW material, assuming that the freezing behavior of the pure substances remains unchanged, even when they are mixed. As already mentioned, the $\mu_{\theta}$ and $\sigma_{\theta}$ values of the pure substances are independent of the particle size. In contrast to that the parameters $\lambda_{\alpha}, \lambda_{\beta}$ and $\lambda_{\text {illite }}$ change when the particle size changes.

From the VH-TDMA measurements we know that the soluble volume fraction of the BPWW material in the internally mixed particle is approximately $9.7 \%$. As mentioned above it might be that the BPWW material on the illite-NX material does not fully dissolve during the few seconds in LACIS, where the particle is immersed in a droplet. In the following we want to discuss three different behaviors which the internally mixed particles may show during the freezing experiments (Fig. 6). Case (a) depicts an extreme case, where 

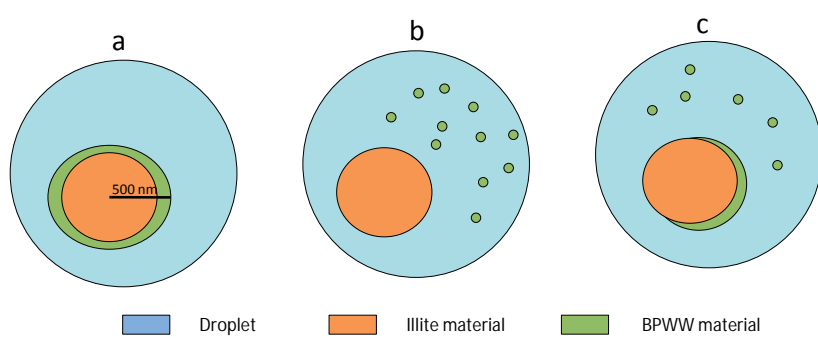

Figure 6. Theoretical forms of mixing of a spherical internally mixed illite-BPWW particle. (a) The illite-NX particle is completely covered with BPWW material which does not dissolve in the droplet. (b) The BPWW material is completely dissolved in the droplet. (c) The BPWW material is partly dissolved and partly still on the illite-NX particle.

the illite-NX particle is fully covered with BPWW material and the BPWW material does not dissolve at all. Only the BPWW material is exposed to the water and can trigger the freezing process. In this case the internally mixed particles would behave exactly like pure $500 \mathrm{~nm}$ BPWW particle (green line in Fig. 5). Obviously this case overestimates the measured ice fractions of the illite-BPWW particles. Case (b) shows another extreme case. Here the whole BPWW material is dissolved in the droplet. Assuming the volume fraction of BPWW material on the illite-BPWW particles to be $9.7 \%$, we can calculate the $\lambda$ values of the BPWW material (assuming $\lambda$ to be volume dependent) as well as $\lambda_{\text {illite }}$ for the remaining spherical illite-NX particle. The values are given in Table 2 . The resulting ice fraction curve is shown in Fig. 5 as a grey line. It is obvious that in this case the ice fraction of the illite-BPWW particles is underestimated. We can conclude from this that the real case is an intermediate case of (a) and (b) (panel c in Fig. 6). The BPWW material dissolves partly, as it was already suggested in Augustin et al. (2013). For this case the size parameters can not be calculated directly as we have no information of how much of the BPWW material will dissolve and how much will still cover the illite-NX core. So we fit Eq. (7) to the measured data with $\lambda_{\alpha}, \lambda_{\beta}$ and $\lambda_{\text {illite }}$ being the fit parameters, where the relation between $\lambda_{\alpha}$ and $\lambda_{\beta}$ remains the same as derived from the measurements of the pure BPWW. This leads to the following results:

$\lambda_{\alpha}=0.206, \lambda_{\beta}=0.041, \lambda_{\text {illite }}=0.511$.

This is depicted as purple line in Fig. 5, and fits the course of the measured data well over the whole temperature range. It should be noted at this point that in the model the ice nucleation properties $\left(\mu_{\theta}\right.$ and $\left.\sigma_{\theta}\right)$ of both materials have not been changed and still are the same as for the pure materials. So with the modeled ice fraction curves we could show that the course of the immersion freezing behavior of the mixture can be explained by the freezing abilities of the pure substances. This can be interpreted as a confirmation of our assumption that it is the ice nucleating active biological fraction which determines the INM containing mixed particles' ice nucleation ability.

\section{Conclusions}

Several studies showed that mineral dust particles can act as carriers for biological material. Up to now it was not shown clearly how a single particle which consists of both mineral dust and biological material behaves in terms of ice nucleation.

In this study we showed that it is possible to quantitatively describe the freezing behavior of particles generated from an illite-BPWW suspension, based on parameters (mean and standard deviation of the contact angle distribution) of the pure substances. In other words, the freezing behavior of the mixed particles appears as superposition of the single substances' freezing behaviors and for a droplet containing such a mixed particle, the most active freezing entity in the droplet will control the freezing process. For the internally mixed particles presented here, this means that if there is an INM located on the surface of an illite-NX particle, this INM will initiate the freezing of the droplet at much higher temperatures than the pure illite-NX particle.

This study also indicates that it is fairly difficult to determine the mixing state even already of a laboratory generated aerosol. The VH-TDMA measurements strongly suggest that the generated particles are internally mixed. However, the amount of biological material on these particles was estimated to be less than $10 \%$ of the whole particle mass. The microscope techniques (SEM and EDX) apparently did not detect this small biological fraction of a mixed bio-dust particle because of their limited analytical possibilities. Also the single particle mass spectrometry (SPLAT) did not detect such small amounts of biological material on all particles. As a result the amount of internally mixed particles appears to be underestimated. This may also be the case for atmospheric measurements. Therefore it can not be ruled out that ice nucleation attributed to mineral dust in the past occasionally might have been due to an undetected biological component.

Based on our results the following advice can be given for the modeling of atmospheric ice nucleation: on the one hand it is not necessary to define new parameterizations for dustbio-mixtures as these particles, depending on actual composition, induce freezing similar to the pure substances. On the other hand, this implies that for proper modeling, the knowledge concerning the number of mineral and biological INP and their mixing state is highly desirable. Finding the respective atmospherically relevant values is still a big challenge as the particle characterization methods are limited in their ability to detect small amounts of certain substances. 
Acknowledgements. This work is partly funded by the German Research Foundation (DFG projects WE 4722/1-1, WE 4722/1-2, EB 383/3-1, EB 383/3-2, SCHN 1138/2-1 and SCHN 1138/2-2) within the DFG Research Unit FOR 1525 INUIT. We thank Dennis Niedermeier for discussions. We also thank the IOM and Andrea Prager for the SEM images. J. Schneider and S. Schmidt thank Oliver Appel for support during the SPLAT measurements.

Edited by: A. Bertram

\section{References}

Atkinson, J. D., Murray, B. J., Woodhouse, M. T., Whale, T. F., Baustian, K. J., Carslaw, K. S., Dobbie, S., O'Sullivan, D., and Malkin, T. L.: The importance of feldspar for ice nucleation by mineral dust in mixed-phase clouds, Nature, 498, 355-358, doi:10.1038/nature12278, 2013.

Augustin, S., Wex, H., Niedermeier, D., Pummer, B., Grothe, H., Hartmann, S., Tomsche, L., Clauss, T., Voigtländer, J., Ignatius, K., and Stratmann, F.: Immersion freezing of birch pollen washing water, Atmos. Chem. Phys., 13, 10989-11003, doi:10.5194/acp-13-10989-2013, 2013.

Augustin-Bauditz, S., Wex, H., Kanter, S., Ebert, M., Niedermeier, D., Stolz, F., Prager, A., and Stratmann, F.: The immersion mode ice nucleation behavior of mineral dusts: a comparison of different pure and surface modified dusts, Geophys. Res. Lett., 41, 7375-7382, doi:10.1002/2014gl061317, 2014.

Bühl, J., Ansmann, A., Seifert, P., Baars, H., and Engelmann, R.: Toward a quantitative characterization of heterogeneous ice formation with lidar/radar: comparison of CALIPSO/CloudSat with ground-based observations, Geophys. Res. Lett., 40, 4404-4408, doi:10.1002/grl.50792, 2013.

Clauss, T., Kiselev, A., Hartmann, S., Augustin, S., Pfeifer, S., Niedermeier, D., Wex, H., and Stratmann, F.: Application of linear polarized light for the discrimination of frozen and liquid droplets in ice nucleation experiments, Atmos. Meas. Tech., 6, 1041-1052, doi:10.5194/amt-6-1041-2013, 2013.

Conen, F., Morris, C. E., Leifeld, J., Yakutin, M. V., and Alewell, C.: Biological residues define the ice nucleation properties of soil dust, Atmos. Chem. Phys., 11, 9643-9648, doi:10.5194/acp-119643-2011, 2011.

DeMott, P. J., Cziczo, D. J., Prenni, A. J., Murphy, D. M., Kreidenweis, S. M., Thomson, D. S., Borys, R., and Rogers, D. C.: Measurements of the concentration and composition of nuclei for cirrus formation, P. Natl. Acad. Sci. USA, 100, 14655-14660, doi:10.1073/pnas.2532677100, 2003.

Fröhlich-Nowoisky, J., Hill, T. C. J., Pummer, B. G., Yordanova, P., Franc, G. D., and Pöschl, U.: Ice nucleation activity in the widespread soil fungus Mortierella alpina, Biogeosciences, 12, 1057-1071, doi:10.5194/bg-12-1057-2015, 2015.

Gysel, M., McFiggans, G. B., and Coe, H.: Inversion of tandem differential mobility analyser (TDMA) measurements, J. Aerosol Sci., 40, 134-151, doi:10.1016/j.jaerosci.2008.07.013, 2009.

Hartmann, S., Niedermeier, D., Voigtländer, J., Clauss, T., Shaw, R. A., Wex, H., Kiselev, A., and Stratmann, F.: Homogeneous and heterogeneous ice nucleation at LACIS: operating principle and theoretical studies, Atmos. Chem. Phys., 11, 17531767, doi:10.5194/acp-11-1753-2011, 2011.
Hartmann, S., Augustin, S., Clauss, T., Wex, H., Šantl-Temkiv, T., Voigtländer, J., Niedermeier, D., and Stratmann, F.: Immersion freezing of ice nucleation active protein complexes, Atmos. Chem. Phys., 13, 5751-5766, doi:10.5194/acp-13-5751-2013, 2013.

Hiranuma, N., Augustin-Bauditz, S., Bingemer, H., Budke, C., Curtius, J., Danielczok, A., Diehl, K., Dreischmeier, K., Ebert, M., Frank, F., Hoffmann, N., Kandler, K., Kiselev, A., Koop, T., Leisner, T., Möhler, O., Nillius, B., Peckhaus, A., Rose, D., Weinbruch, S., Wex, H., Boose, Y., DeMott, P. J., Hader, J. D., Hill, T. C. J., Kanji, Z. A., Kulkarni, G., Levin, E. J. T., McCluskey, C. S., Murakami, M., Murray, B. J., Niedermeier, D., Petters, M. D., O’Sullivan, D., Saito, A., Schill, G. P., Tajiri, T., Tolbert, M. A., Welti, A., Whale, T. F., Wright, T. P., and Yamashita, K.: A comprehensive laboratory study on the immersion freezing behavior of illite NX particles: a comparison of 17 ice nucleation measurement techniques, Atmos. Chem. Phys., 15, 2489-2518, doi:10.5194/acp-15-2489-2015, 2015.

Kamphus, M., Ettner-Mahl, M., Brands, M., Curtius, J., Drewnick, F., and Borrmann, S.: Comparison of two aerodynamic lenses as an inlet for a single particle laser ablation mass spectrometer, Aerosol Sci. Tech., 42, 970-980, doi:10.1080/02786820802372158, 2008.

Kanitz, T., Seifert, P., Ansmann, A., Engelmann, R., Althausen, D., Casiccia, C., and Rohwer, E. G.: Contrasting the impact of aerosols at northern and southern midlatitudes on heterogeneous ice formation, Geophys. Res. Lett., 38, L17802, doi:10.1029/2011g1048532, 2011.

Kleber, M., Sollins, P., and Sutton, R.: A conceptual model of organo-mineral interactions in soils: self-assembly of organic molecular fragments into zonal structures on mineral surfaces, Biogeochemistry, 85, 9-24, doi:10.1007/s10533-0079103-5, 2007.

Knutson, E. O. and Whitby, K. T.: Aerosol classification by electric mobility: apparatus, theory, and applications, J. Aerosol Sci., 6, 443-451, doi:10.1016/0021-8502(75)90060-9, 1975.

Kumai, M.: Snow crystals and the identification of the nuclei in the northern United-States of America, J. Meteorol., 18, 139-150, doi:10.1175/1520-0469(1961)018<0139:scatio>2.0.co;2, 1961.

Maki, L. R., Galyan, E. L., Chang-Chien, M., and Caldwell, D. R.: Ice nucleation induced by pseudomonas-syringea, Appl. Microbiol., 28, 456-459, 1974.

Michaud, A. B., Dore, J. E., Leslie, D., Lyons, W. B., Sands, D. C., and Priscu, J. C.: Biological ice nucleation initiates hailstone formation, J. Geophys. Res.-Atmos., 119, 12186-12197, doi:10.1002/2014jd022004, 2014.

Morris, C. E., Sands, D. C., Vinatzer, B. A., Glaux, C., Guilbaud, C., Buffiere, A., Yan, S., Dominguez, H., and Thompson, B. M.: The life history of the plant pathogen Pseudomonas syringae is linked to the water cycle, Isme J., 2, 321-334, doi:10.1038/ismej.2007.113, 2008.

Murray, B. J., O'Sullivan, D., Atkinson, J. D., and Webb, M. E.: Ice nucleation by particles immersed in supercooled cloud droplets, Chem. Soc. Rev., 41, 6519-6554, doi:10.1039/c2cs35200a, 2012.

Niedermeier, D., Ervens, B., Clauss, T., Voigtländer, J., Wex, H., Hartmann, S., and Stratmann, F.: A computationally efficient description of heterogeneous freezing: a simplified version 
of the Soccerball model, Geophys. Res. Lett., 41, 736-741, doi:10.1002/2013g1058684, 2014.

Niedermeier, D., Augustin-Bauditz, S., Hartmann, S., Wex, H., Ignatius, K., and Stratmann, F.: Can we define an asymptotic value for the ice active surface site density for heterogeneous ice nucleation?, J. Geophys. Res.-Atmos., 120, 50365046, doi:10.1002/2014JD022814, 2015.

O'Sullivan, D., Murray, B. J., Malkin, T. L., Whale, T. F., Umo, N. S., Atkinson, J. D., Price, H. C., Baustian, K. J., Browse, J., and Webb, M. E.: Ice nucleation by fertile soil dusts: relative importance of mineral and biogenic components, Atmos. Chem. Phys., 14, 1853-1867, doi:10.5194/acp-14-18532014, 2014.

Petters, M. D. and Kreidenweis, S. M.: A single parameter representation of hygroscopic growth and cloud condensation nucleus activity, Atmos. Chem. Phys., 7, 1961-1971, doi:10.5194/acp-71961-2007, 2007.

Pratt, K. A., DeMott, P. J., French, J. R., Wang, Z., Westphal, D. L., Heymsfield, A. J., Twohy, C. H., Prenni, A. J., and Prather, K. A.: In situ detection of biological particles in cloud ice-crystals, Nat. Geosci., 2, 397-400, doi:10.1038/ngeo521, 2009.

Pummer, B. G., Bauer, H., Bernardi, J., Bleicher, S., and Grothe, H.: Suspendable macromolecules are responsible for ice nucleation activity of birch and conifer pollen, Atmos. Chem. Phys., 12, 2541-2550, doi:10.5194/acp-12-2541-2012, 2012.

Pummer, B. G., Budke, C., Augustin-Bauditz, S., Niedermeier, D., Felgitsch, L., Kampf, C. J., Huber, R. G., Liedl, K. R., Loerting, T., Moschen, T., Schauperl, M., Tollinger, M., Morris, C. E., Wex, H., Grothe, H., Pöschl, U., Koop, T., and FröhlichNowoisky, J.: Ice nucleation by water-soluble macromolecules, Atmos. Chem. Phys., 15, 4077-4091, doi:10.5194/acp-15-40772015, 2015.

Schnell, R. C. and Vali, G.: Biogenic ice nuclei: Part I. Terrestrial and marine sources, J. Atmos. Sci., 33, 1554-1564, doi:10.1175/1520-0469(1976)033<1554: BINPIT>2.0.CO;2, 1976.
Seifert, P., Ansmann, A., Mattis, I., Wandinger, U., Tesche, M., Engelmann, R., Mueller, D., Perez, C., and Haustein, K.: Saharan dust and heterogeneous ice formation: eleven years of cloud observations at a central European EARLINET site, J. Geophys. Res.-Atmos., 115, D20201, doi:10.1029/2009jd013222, 2010.

Stratmann, F., Kiselev, A., Wurzler, S., Wendisch, M., Heintzenberg, J., Charlson, R. J., Diehl, K., Wex, H., and Schmidt, S.: Laboratory studies and numerical simulations of cloud droplet formation under realistic supersaturation conditions, J. Atmos. Ocean. Tech., 21, 876-887, doi:10.1175/15200426(2004)021<0876:1sanso>2.0.co;2, 2004.

Szyrmer, W. and I. Zawadzki: Biogenic and anthropogenic sources of ice forming nuclei: A review, B. Am. Meteorol. Soc., 78, 209228, 1997.

Tobo, Y., DeMott, P. J., Hill, T. C. J., Prenni, A. J., SwobodaColberg, N. G., Franc, G. D., and Kreidenweis, S. M.: Organic matter matters for ice nuclei of agricultural soil origin, Atmos. Chem. Phys., 14, 8521-8531, doi:10.5194/acp-14-8521-2014, 2014.

Wilson, T. W., Ladino, L. A., Alpert, P. A., Breckels, M. N., Brooks, I. M., Browse, J., Burrows, S. M., Carslaw, K. S., Huffman, J. A., Judd, C., Kilthau, W. P., Mason, R. H., McFiggans, G., Miller, L. A., Najera, J. J., Polishchuk, E., Rae, S., Schiller, C. L., Si, M., Temprado, J. V., Whale, T. F., Wong, J. P. S., Wurl, O., Yakobi-Hancock, J. D., Abbatt, J. P. D., Aller, J. Y., Bertram, A. K., Knopf, D. A., and Murray, B. J.: A marine biogenic source of atmospheric ice-nucleating particles, Nature, 525, 234-238, doi:10.1038/nature14986, 2015.

Wolber, P. K., Deininger, C. A., Southworth, M. W., Vandekerckhove, J., Vanmontagu, M., and Warren, G. J.: Identification and purification of a bacterial ice-nucleation protein, P. Natl. Acad. Sci. USA, 83, 7256-7260, doi:10.1073/pnas.83.19.7256, 1986.

Zobrist, B., Koop, T., Luo, B. P., Marcolli, C., and Peter, T.: Heterogeneous ice nucleation rate coefficient of water droplets coated by a nonadecanol monolayer, J. Phys. Chem.-US, 111, 21492155, doi:10.1021/jp066080w, 2007. 